\title{
How many detector pixels do we need for super-resolution ptychography?
}

Xiyue Zhang ${ }^{1}$, Zhen Chen $^{2}$ and David Muller ${ }^{3}$

${ }^{1}$ Cornell University, Ithaca, New York, United States, ${ }^{2}$ School of Applied and Engineering Physics, Cornell University, Ithaca, NY, USA, New York, United States, ${ }^{3}$ School of Applied and Engineering Physics, Cornell University, Ithaca, New York, United States

In traditional scanning transmission electron microscopy (STEM) imaging, the spatial resolution limit is set by the diffraction limit from the probe-forming aperture. This limit can be overcome by ptychography, where we collect a 4-dimensional dataset consisting of a diffraction pattern ( $\mathrm{kx}, \mathrm{ky})$ at each scan position $(\mathrm{x}, \mathrm{y})$ for phase reconstruction. With the development of iterative phase retrieval algorithms such as ePIE and high dynamical range pixel-array detectors that enable the acquisition of the full scattering distribution, images with usable information beyond the diffraction limit have been achieved [1]. One challenge for ptychography is the relatively slow acquisition speed of the 4D dataset $-0.1-1 \mathrm{~ms} / \mathrm{real}$ space position compared to 0.1-10 $\mu$ s for differential phase contrast (DPC) imaging with a quadrant i.e. (2x2) detector. The longer acquisition times lead to more noticeable scan noise, drift and damage. Here, we demonstrate that super-resolution ptychography is still possible with only $2 \times 2$ detector pixels when the real space sampling is sufficient and that the ptychography can significantly outperform the conventional DPC analysis. Smaller detectors such as segmented detectors are much faster thus we now open up the possibility of using such faster detectors for ptychography[2].

The ptychographic sampling ratio $\mathrm{Sx}, \mathrm{y}=1 /(\mathrm{UR})$, where $\mathrm{U}$ is the reciprocal space sampling and $\mathrm{R}$ is the real space sampling, is the criterion that determines the quality of electron ptychography phase reconstruction [3]. For the same quality of reconstruction, we can trade-off the reciprocal space sampling for increased real space sampling. Given fewer detector pixels, to compensate for the diffraction patterns undersampling with respect to Nyquist detector sampling, an oversampling in real space is therefore required. We explored the effect of Sx,y on the resolution by varying the probe scan step size and the detector-pixel number in the simulated 4D-STEM datasets. Figure 1 shows that diffraction datasets with more than $8 \times 8$ detector pixels still give good reconstructions, but when the diffraction dataset has only $4 \times 4$ detector pixels, the reconstructed image contrast is degraded.

However, as $\mathrm{Sx}, \mathrm{y}=8$ is still large, a reconstruction should still be possible. In fact, a reciprocal space upsampling in the phase retrieval processing is very useful, because it helps to access the data redundancy from probe positions in the object plane, provided $\mathrm{Sx}, \mathrm{y}>>1$ [4]. Virtual detector subpixels can be enabled for undersampled diffraction patterns by interpolating the diffraction patterns and normalizing intensity within each original detector pixel after the estimate of the exit wave in each iteration of ePIE [4]. Using subpixel upsampling in ePIE, a good phase reconstruction from $2 \times 2$ detector pixels is accomplished (Fig. 1f), which otherwise would fail to give meaningful structures.

A resolution comparison between conventional integrated DPC (iDPC) [5] and ptychography, both using the same $2 \times 2$ detector pixels is displayed in figure 2 . For the same $2 \times 2$ detector dataset, ptychography performs better in resolving the Si dumbbell structure - both in terms of FWHM and dip between adjacent atoms, which means 4 detector-pixels are sufficient to maintain super-resolution ptychography. The 
significant reduction of pixel numbers enables the usage of fast segmented detectors for ptychography. [7]
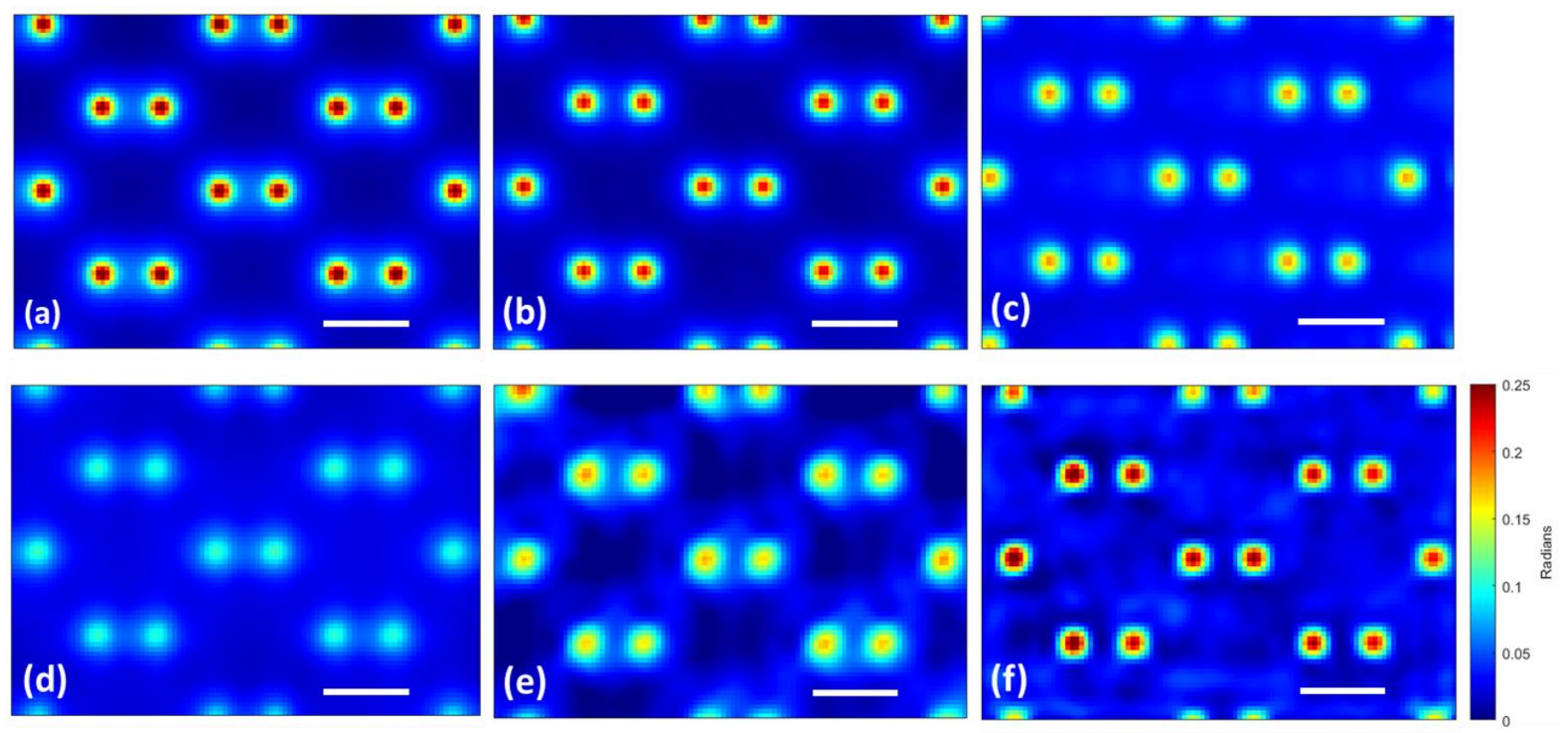

Figure 1. Figure 1. 4D-STEM ptychographical phase retrieval of Si (110) dumbbell structure by ePIE as a function of detector pixels. (a) Reconstruction from $64 \times 64$ detector-pixels with $\mathrm{Sx}, \mathrm{y}=132$ has excellent resolution. (b) Reconstruction from $16 \times 16$ detector-pixels with Sx,y = 33 still has good resolution. (c) Image contrast reconstructed from $8 \times 8$ detector-pixels with Sx,y $=17$ has degraded. (d) Image contrast reconstructed from $4 \times 4$ detector-pixels with $\mathrm{Sx}, \mathrm{y}=8$ has further degraded. (e) With 16 times upsampling to $64 \times 64$ pixels, image contrast reconstructed from $4 \times 4$ detector-pixels with Sx,y $=8$ has only minor artifacts. (f) With 32 times upsampling to $64 \times 64$ detector pixels, image contrast reconstructed from only $2 \times 2$ detector-pixels $(\mathrm{Sx}, \mathrm{y}=4)$ is recovered. All simulated 4D-STEM datasets have the same probe scan step size $0.102 \AA$ but a different number of detector pixels. The 4D-STEM dataset was simulated at $300 \mathrm{kV}$ beam voltage, $24 \mathrm{mrad}$ convergence angle $\alpha$ and Scherzer defocus with $0.01 \mathrm{~mm}$ spherical aberration, on the single-unit cell thickness $\mathrm{Si}$ (110) plane with $2 \alpha$ maximum scattering angle by $\mu \mathrm{STEM}[6]$. The scalebar is $2 \AA$ and colormap is the same for all the images. 
(a) Integrated differential phase contrast

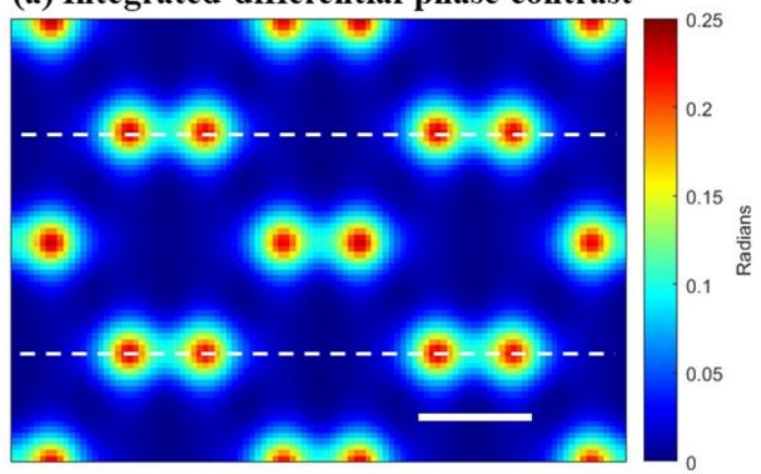

(c) Line profile from iDPC

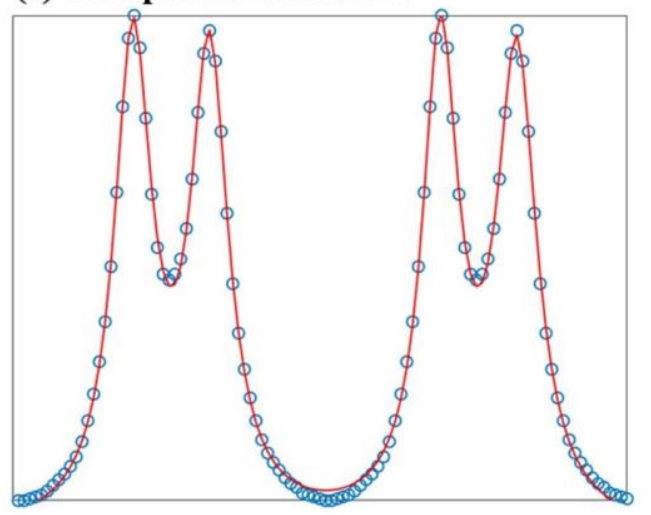

(b) ePIE reconstruction

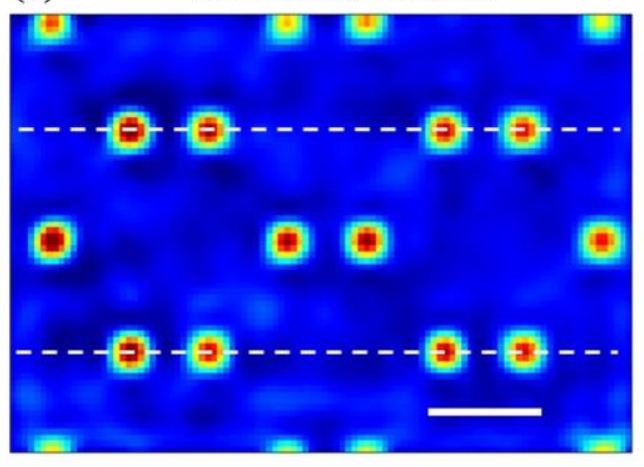

(d) Line profile from ePIE

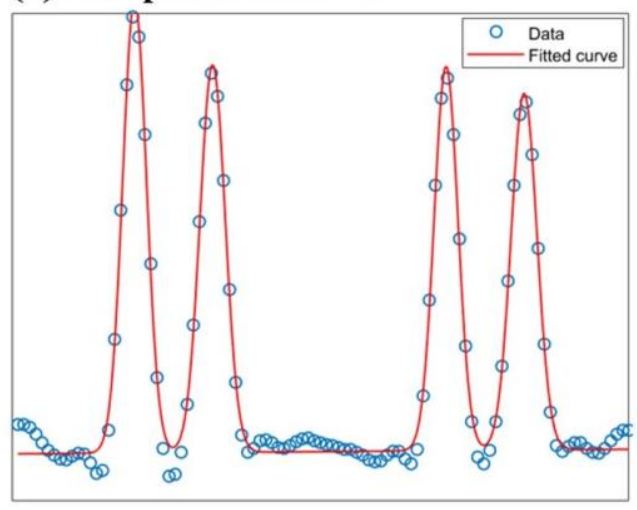

Figure 2. Figure 2: Phase contrast images of $\mathrm{Si}$ (110) dumbbell structure reconstructed by integrated differential phase contrast (iDPC) and ptychographical iterative engine (ePIE) using the same $2 \times 2$ pixels detector 4D-STEM dataset. Fig. 2c\& $2 d$ ) show line profiles at the position of white dashed lines in Fig. $2 \mathrm{a} \& 2 \mathrm{~b}$. With an only $2 \times 2$ pixels detector, ptychography with the reciprocal space upsampling outperforms iDPC with 1.6 times better resolution by comparing the full widths at half maximum (FWHM) -0.51 vs $0.80 \AA$. This is also reflected by the depth of dip between the 2 atoms in the $1.36 \AA$ dumbbell structure: Ptychography cleanly resolves this dip at almost $100 \%$, which surpasses the $56 \%$ dip from iDPC. The scalebar is $2 \AA$ and the colorbar is the same for both images.

\section{References}

[1] Y. Jiang, Z. Chen et al., Electron ptychography of 2D materials to deep sub-angstrom resolution, Nature 559(2018), 343-349.

[2] H.G. Brown et al., Structure retrieval with fast electrons using segmented detectors, Physical Review B 93(2016), 134116.

[3] T.B. Edo et al., Sampling in x-ray Ptychography, Physical Review A 87(2013), 053850.

[4] D.J. Batey et al., Reciprocal-space up-sampling from real-space oversampling in x-ray ptychography, Physical Review A 89(2014), 043812.

[5] R. Close et al., Towards quantitative, atomic-resolution reconstruction of the electrostatic potential via differential phase contrast using electrons, Ultramicroscopy 159(2015), 124-137.

[6] L.J. Allen et al., Modelling the inelastic scattering of fast electrons, Ultramicroscopy, 151(2015), 1122.

[7] Work supported by US NSF (grants DMR-1719875 and DMR-1539918) 